\title{
INTEGRAL BROWN-GITLER SPECTRA
}

\author{
FRED R. COHEN, DONALD M. DAVIS, PAUL G. GOERSS
}

AND MARK E. MAHOWALD

(Communicated by Haynes R. Miller)

\begin{abstract}
A Thom spectrum model for integral Brown-Gitler spectra is established and shown to have a multiplicative property. This clarifies certain aspects of an earlier application to splitting $b o \wedge b o$.
\end{abstract}

1. Statement of results. Brown-Gitler spectra have had many important applications in homotopy theory, most notably in [M1 and C1]. They were originally constructed in [BG] by a complicated Postnikov argument, but a Thom spectrum model suggested in [M1] and established to be correct in [C2] made them more down-to-earth.

Integral Brown-Gitler spectra at the prime 2 were introduced in [M2], where they were useful in a splitting of $b o \wedge b o$. A Thom spectrum model was suggested there, and an expanded account, including both Thom spectrum and Postnikov models, was presented in [Sh]. The odd-primary version of the Thom space model was discussed in [Ka]. In none of these is the base space for these Thom spectra explicitly defined. The purpose of this paper is to clarify the Thom spectrum model of integral Brown-Gitler spectra.

Recall that there is an isomorphism of Hopf algebras

$$
H_{*}\left(\Omega^{2} S^{3}\right) \approx E\left[x_{j}: j \geq 0\right] \otimes \mathbf{F}_{p}\left[y_{j}: j \geq 1\right] \quad \text { if } p \text { odd }
$$

with $\left|x_{j}\right|=2 p^{j}-1$ and $\left|y_{j}\right|=2 p^{j}-2$. The only modification required for $p=2$ is $x_{j}^{2}=y_{j+1}$. All homology groups have coefficients in the field $\mathbf{F}_{p}$ with $p$ elements, unless indicated otherwise. Define a weight on the monomials in $H_{*}\left(\Omega^{2} S^{3}\right)$ by

$$
\operatorname{wt}\left(x_{j}\right)=\operatorname{wt}\left(y_{j}\right)=p^{j}, \quad \operatorname{wt}(a b)=\operatorname{wt}(a)+\operatorname{wt}(b) .
$$

The space $\Omega^{2} S^{3}$ admits an increasing filtration by spaces $F_{n} \Omega^{2} S^{3}$, due to May and Milgram [May, Mil], such that $H_{*}\left(F_{n} \Omega^{2} S^{3}\right) \subset H_{*}\left(\Omega^{2} S^{3}\right)$ is the span of monomials of weight $\leq n$ [CLM, p. 239].

Let $S^{3}\langle 3\rangle$ denote the 3-connected cover of $S^{3}$. Then there is a homotopy fibration

$$
\Omega^{2} S^{3}\langle 3\rangle \rightarrow \Omega^{2} S^{3} \rightarrow S^{1} .
$$

$\Omega^{2} S^{3}\langle 3\rangle$ was called $W$ in [DGM and M2]. Using the multiplication on $\Omega^{2} S^{3}$, one easily deduces $\Omega^{2} S^{3} \simeq S^{1} \times \Omega^{2} S^{3}\langle 3\rangle$, and so $H_{*}\left(\Omega^{2} S^{3}\langle 3\rangle\right) \subset H_{*}\left(\Omega^{2} S^{3}\right)$ is the span

Received by the editors October 20, 1987.

1980 Mathematics Subject Classification (1985 Revision). Primary 55P42, 55P35.

Key words and phrases. Brown-Gitler spectra, Thom spectra, loop spaces.

All authors were supported by National Science Foundation research grants. 
of monomials of weight divisible by $p$. The filtration on $\Omega^{2} S^{3}$ induces a filtration on $H_{*}\left(\Omega^{2} S^{3}\langle 3\rangle\right)$ by

$$
F_{n} H_{*} \Omega^{2} S^{3}\langle 3\rangle=H_{*}\left(F_{n} \Omega^{2} S^{3}\right) \cap H_{*}\left(\Omega^{2} S^{3}\langle 3\rangle\right),
$$

the span of monomials of weight $\leq n$ and divisible by $p$. In [M2] for $p=2$ and [Ka] for odd $p$, it was asserted without proof that $F_{n} H_{*} \Omega^{2} S^{3}\langle 3\rangle$ is induced by an actual filtration of the space $\Omega^{2} S^{3}\langle 3\rangle$. This does not follow for general reasons, but we shall show that it can be achieved after localization with respect to mod $p$ homology.

We denote by $X_{p}$ the Bousfield localization [Bo] of the space $X$ with respect to the homology theory $H_{*}\left(-; \mathbf{F}_{p}\right)=H \mathbf{F}_{p *}$. For a fixed prime $p$, let $\mathcal{F}_{n}=\left(F_{n} \Omega^{2} S^{3}\right)_{p}$. There are product maps

$$
\mathcal{F}_{m} \times \mathcal{F}_{n} \stackrel{\mu}{\rightarrow} \mathcal{F}_{m+n}
$$

induced by the corresponding maps for the filtration spaces and the fact that localization preserves finite products $[\mathrm{Bo}, 12.5]$. Define $A_{n}$ by the homotopy fibration sequence

$$
A_{n} \rightarrow \mathcal{F}_{p n+1} \rightarrow S_{p}^{1}
$$

where the second map is the localization of the composite

$$
F_{p n+1} \Omega^{2} S^{3} \rightarrow \Omega^{2} S^{3} \rightarrow S^{1} .
$$

It follows easily from the definitions and [Bo, 12.7] that the space $A_{n}$ is $H \mathbf{F}_{p *}$-local.

Most of our effort is directed toward the following result, which is proved in $\S 2$.

THEOREM 1.3. The fiber sequence (1.2) is equivalent to a product fibration. Indeed, there is a map $A_{n} \stackrel{\phi}{\longrightarrow} \mathcal{F}_{p n}$ and a commutative diagram of fibrations

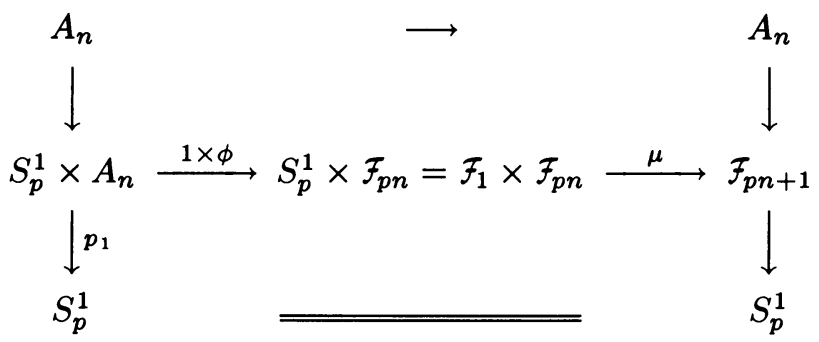

which is an equivalence on total spaces and on fibers.

Then $H_{*}\left(A_{n}\right) \approx F_{p n} H_{*}\left(\Omega^{2} S^{3}\langle 3\rangle\right)$, the span of monomials of weight pi with $i \leq n$.

REMARK. That $H_{*} A_{n}$ is as claimed is not immediate from (1.2) and the Serre spectral sequence, since it is not clear a priori that the fibration of (1.2) is orientable. For example, the fibration $\mathcal{F}_{p^{k}} \rightarrow S_{p}^{1}$ is not orientable. Our Theorem 1.3 establishes the orientability in (1.2) indirectly. that

In [M3], Mahowald constructed a stable spherical fibration $\xi$ over $\Omega^{2} S_{p}^{3}$ such

(i) the Thom spectrum $T(\xi)$ is equivalent to the $\bmod p$ Eilenberg-Mac Lane spectrum $H \mathrm{Z} / p$,

(ii) the Thom spectrum $T\left(\xi \mid \Omega^{3} S^{3}\langle 3\rangle_{p}\right)$ is equivalent to the $p$-complete Eilenberg-Mac Lane spectrum $H \mathbf{Z}_{p}^{\widehat{p}}$, and 
(iii) the Thom spectrum $T\left(\xi \mid F_{n} \Omega^{2} S_{p}^{3}\right)$ is equivalent to the $n$th $\bmod p$ BrownGitler spectrum $B(n)$.

Expanded accounts appear in [Ka and CMT].

We define $B_{1}(n)$, the $n$th integral Brown-Gitler spectrum at $p$, to be the Thom spectrum $T\left(\xi \mid A_{n}\right)$, using the commutative diagram

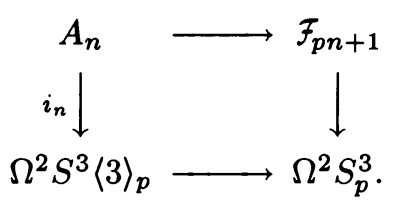

Thomifying the composites

$$
A_{m} \times A_{n} \stackrel{\phi_{m} \times \phi_{n}}{\longrightarrow} \mathcal{F}_{p m} \times \mathcal{F}_{p n} \rightarrow \mathcal{F}_{p m+p n} \rightarrow \mathcal{F}_{p m+p n+1} \rightarrow A_{m+n}
$$

where the last map splits the equivalence of 1.3 , yields pairings $B_{1}(m) \wedge B_{1}(n) \rightarrow$ $B_{1}(m+n)$. These pairings played a crucial role in the application to splitting $b o \wedge b o$ in [DGM, Ka and M2]. The clarification of their existence is a major reason for the care in this work.

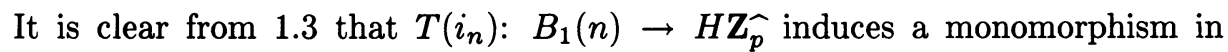
homology. Recall Milnor's description

$$
H_{*}\left(H \mathbf{Z}_{p}\right)=E\left[\chi\left(\tau_{j}\right): j \geq 1\right] \otimes \mathbf{F}_{p}\left[\chi\left(\xi_{j}\right): j \geq 1\right],
$$

where $\left|\tau_{j}\right|=2 p^{j}-1,\left|\xi_{j}\right|=2 p^{j}-2$, and $\chi$ denotes the canonical antiautomorphism of the dual of the mod $p$ Steenrod algebra $A$. The usual modification $\tau_{j}^{2}=\xi_{j+1}$ applies when $p=2$. We define a weight by

$$
\operatorname{wt}\left(\chi\left(\tau_{j}\right)\right)=\operatorname{wt}\left(\chi\left(\xi_{j}\right)\right)=p^{j}, \quad \operatorname{wt}(a b)=\operatorname{wt}(a)+\operatorname{wt}(b) .
$$

Note that all monomials have weight divisible by $p$. The relationship between these classes and those in (1.1) under the Thom isomorphism was discussed in [CMT].

The first two parts of the following theorem, which states the basic properties of integral Brown-Gitler spectra, now follow immediately from Theorem 1.3.

THEOREM 1.5. For $n>0$, there is a $p$-complete spectrum $B_{1}(n)$ and a map

$$
B_{1}(n) \stackrel{g}{\rightarrow} H \mathbf{Z}_{p}^{\wedge}
$$

such that

(i) $g_{*}$ sends $H_{*}\left(B_{1}(n)\right)$ isomorphically onto the span of monomials of weight $\leq p n$

(ii) there are pairings

$$
B_{1}(m) \wedge B_{1}(n) \rightarrow B_{1}(m+n)
$$

whose homology homomorphism is compatible with the multiplication in $H_{*}\left(H \mathbf{Z}_{p}\right)$;

(iii) for any $C W$ complex $X$,

$$
g_{*}: B_{1}(n)_{i}(X) \rightarrow H_{i}\left(X ; \mathbf{Z}_{p}^{\widehat{p}}\right)
$$

is surjective if $i \leq 2 p(n+1)-1$. 
Part (iii) is not easily proved from our perspective, but does follow from a straightforward adaptation of the proof of [Sh, 5.1], which was given only for $p=2$. This adaptation requires a map from $B_{1}(n)$ into the $\bmod p$ BrownGitler spectrum $B(p n+1)$ inducing the obvious inclusion in homology. Such a map follows immediately from the definitions and (1.4). The argument of [Sh] then allows us to deduce the surjectivity of $B_{1}(n)_{*}(X) \rightarrow H_{*}\left(X ; \mathbf{Z}_{p}\right)$ from that of $B(p n+1)_{*}(X) \rightarrow H_{*}\left(X ; \mathbf{F}_{p}\right)$.

Many readers may be more familiar with the cohomology criterion

$$
H^{*}\left(B_{1}(n)\right) \approx A / A\left(\beta, \chi P^{i}: i>n\right) .
$$

This is easily seen to be dual to (i) above. We also remark that our indexing differs from that of [Sh and $\mathbf{K a}$ ], who would call our spectrum $B_{1}(p n+1)$. We thank Don Shimamoto for helpful comments.

2. Proof of Theorem 1.3. We begin by showing that the inclusion $F_{m-1} \Omega^{2} S^{3}$ $\rightarrow F_{m} \Omega^{2} S^{3}$ may be considered as the inclusion into a mapping cone. Let $\Sigma_{m}$ denote the symmetric group on $m$ letters, and $F\left(\mathbf{R}^{2}, m\right)$ the space of $m$-tuples of distinct points in $\mathbf{R}^{2}$. If $X$ is a pointed $\Sigma_{m}$-space, we define

$$
M_{m}(X)=F\left(\mathbf{R}^{2}, m\right) \times_{\Sigma_{m}} X / F\left(\mathbf{R}^{2}, m\right) \times_{\Sigma_{m}} * .
$$

Let $I$ denote the unit interval, $I$ its boundary, and $\partial I^{m}$ the boundary of $I^{m}$.

LEMMA 2.1. Let $F_{m}=F_{m} \Omega^{2} S^{3}$. There is a cofibration sequence

$$
M_{m}\left(\partial I^{m}\right) \stackrel{c}{\rightarrow} F_{m-1} \rightarrow F_{m}
$$

REMARK. Extending this cofibration shows that $\Sigma M_{m}\left(\partial I^{m}\right) \simeq F_{m} / F_{m-1}$. In particular, $H_{*}\left(M_{m}\left(\partial I^{m}\right)\right)$ is clear from the cofibration.

PROOF. Let $T^{m}(I / \dot{I})$ denote the fat wedge, consisting of points in the $m$-fold Cartesian product having at least one component the basepoint. Viewing $I^{m}$ as the cone $C\left(\partial I^{m}\right)$ yields a $\Sigma_{m}$-equivariant cofibration

$$
\partial I^{m} \rightarrow T^{m}(I / \dot{I}) \hookrightarrow(I / \dot{I})^{\times m}
$$

and hence a cofibration

$$
M_{m}\left(\partial I^{m}\right) \stackrel{k}{\rightarrow} M_{m}\left(T^{m}(I / \dot{I})\right) \rightarrow M_{m}\left((I / \dot{I})^{\times m}\right) .
$$

Recall from [May] that

$$
F_{m}=\left(\bigcup_{k \leq m} M_{k}\left((I / \dot{I})^{\times k}\right)\right) / \sim,
$$

where, letting ` denote omission,

$$
\left(x_{1}, \ldots, x_{k}, t_{1}, \ldots, t_{k}\right) \sim\left(x_{1}, \ldots, \hat{x}_{i}, \ldots, x_{k}, t_{i}, \ldots, \hat{t}_{i}, \ldots, t_{k}\right) \text { if } t_{i}=* \in I / \dot{I}
$$

The map $c$ of Lemma 2.1 is the composite

$$
M_{m}\left(\partial I^{m}\right) \rightarrow M_{m}\left(T^{m}(I / \dot{I})\right) \rightarrow F_{m-1},
$$

where the second map uses the equivalence relation (2.3) to ignore the basepoint in at least one component. The required homeomorphism from the mapping cone 
of $c$ to $F_{m}$ is a quotient of the homeomorphism in (2.2) from the mapping cone of $k$ to $M_{m}\left((I / \dot{I})^{\times m}\right)$.

Now we return to the proof of Theorem 1.3. Assume by induction that the theorem has been proved for $n-1$. Note that $\mathcal{F}_{p(n-1)+1} \rightarrow \mathcal{F}_{p n-1}$ is an equivalence. Localizing Lemma 2.1 yields a map

$$
M_{p n}\left(\partial I^{p n}\right)_{p} \rightarrow \xi_{p n-1} \simeq S_{p}^{1} \times A_{n-1},
$$

and, since $H^{1}\left(M_{p n}\left(\partial I^{p n}\right)_{p} ; \mathbf{Z}_{p}\right)=0$, the map is of the form $* \times h$. Let $Y$ denote the mapping cone of $h$. The map of cofibrations

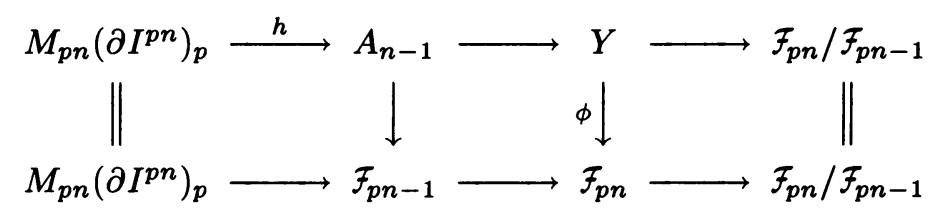

shows that $H_{*} Y \rightarrow H_{*} \mathcal{F}_{p n}$ is injective with image spanned by monomials of weight divisible by $p$. There is a map of fibrations

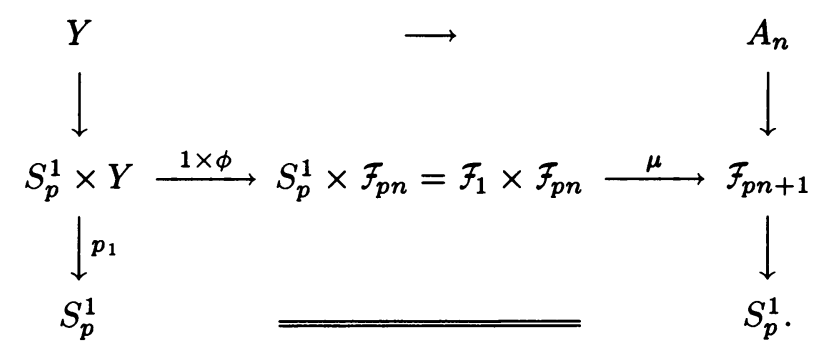

The map of total spaces induces an isomorphism in $\bmod p$ homology, and, since $\mathcal{F}_{p n+1}$ is $H \mathbf{F}_{p *}$-local, this map is an $H \mathbf{F}_{p *}$-localization, and so there is an equivalence of fibrations

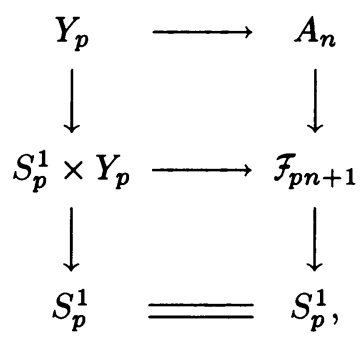

extending the induction, and completing the proof of Theorem 1.3.

\section{REFERENCES}

[Bo] A. K. Bousfield, The localization of spaces with respect to homology, Topology 14 (1975), 133150.

[BG] E. H. Brown and S. Gitler, A spectrum whose cohomology is a certain cyclic module over the Steenrod algebra, Topology 12 (1973), 283-295.

[CLM] F. R. Cohen, T. J. Lada and J. P. May, The homology of iterated loop spaces, Lecture Notes in Math., vol. 533, Springer- Verlag, 1976.

[CMT] F. R. Cohen, J. P. May and L. Taylor, $K(\mathbf{Z}, 0)$ and $K\left(\mathbf{Z}_{2}, 0\right)$ as Thom spectra, Illinois J. Math. 25 (1981), 99-106.

[C1] R. L. Cohen, Immersions of manifolds, Ann. of Math. 122 (1985), 237-328. 
[C2] _ The geometry of $\Omega^{2} S^{3}$ and braid orientations, Invent. Math. 54 (1979), 53-67.

[DGM] D. M. Davis, S. Gitler and M. Mahowald, The stable geometric dimension of vector bundles over real projective spaces, Trans. Amer. Math. Soc. 268 (1981), 39-61.

[Ka] R. M. Kane, Operations in connective K-theory, Mem. Amer. Math. Soc. 254 (1981).

[M1] M. Mahowald, A new infinite family in $\pi_{*}^{3}$, Topology 16 (1977), 249-256.

[M2] _ bo-resolutions, Pacific J. Math. 92 (1981), 365-383.

[M3] _ Ring spectra which are Thom complexes, Duke Math. J. 46 (1979), 549-559.

[May] J. P. May, The geometry of iterated loop spaces, Lecture Notes in Math., vol. 271, SpringerVerlag, 1972.

[Mil] R. J. Milgram, Iterated loop spaces, Ann. of Math. 84 (1966), 386-403.

[Sh] D. H. Shimamoto, An integral version of the Brown-Gitler spectrum, Trans. Amer. Math. Soc. 283 (1984), 383-421.

Department of Mathematics, University of KentuCky, Lexington, KenTUCKY 40506 18015

Department of Mathematics, Lehigh UNiversity, Bethlehem, PenNsylvania

Department of Mathematics, Wellesley College, Wellesley, MassachuSETTS 02181

DEPARTMENT OF MATHEMATICS, NORTHWESTERN UNIVERSITY, EVANSTON, ILliNOIS 60201 